\title{
La lectura en el ámbito universitario. Estrategias para intentar resolver un conflicto.
}

Reading at university level. Strategies for attempting to resolve conflict.
Leitura em universidade level. Estratégias para tentar resolver conflitos.
Fecha de Recepción

14 de mayo de 2014
Aceptado para su publicación

II de septiembre de 2014
Milagros Rojo Guiñazú Chiozzi Profesora Titular Cátedra "Comunicación oral y escrita II", FOUNNE.

Bruno Aldo Ragazzi Auxiliar de $1{ }^{\circ}$ Cátedra "Comunicación oral y escrita II", FOUNNE

Verónica Zibelman Profesora y Licenciada en Letras.

Lugar de Trabajo Facultad de Odontologia - UNNE. Avenida Libertad 5450 (3400). Corrientes. Argentina.

\section{Resumen}

En el siguiente artículo desarrollaremos una reflexión y se propondrán algunas estrategias de resolución vinculadas con una problemática que afecta a la comunidad universitaria: el proceso de lectura académica y la comprensión de los textos.

Pensamos en la comprensión de la lectura de un texto académico como la recuperación de su significado a partir de la consideración de ciertas pistas contenidas en el mismo texto. La mencionada recuperación se lleva a cabo -indudablemente- mediante la ejecución de operaciones mentales que pone en funcionamiento el lector con la finalidad de darle sentido a las pistas halladas. Referimos a un proceso dinámico que desarrolla el que lee, a medida que establece conexiones coherentes entre la información que posee en sus estructuras cognitivas y la nueva información que le provee el texto.

Partiendo de estos postulados teóricos desarrollaremos nuestra propuesta a través de los siguientes tópicos: el proceso de lectura; las fases de la lectura; principales conflictos; y posibles estrategias de resolución.

\section{Palabras Clave \\ Lectura - Estrategias - Proceso - Comprensión Conflicto}




\section{Abstract}

In the following article we will develop some reflection and resolution strategies, linked to a problem that affects the university community will be proposed: the process of reading and understanding academic texts.

We believe in understanding reading an academic text as the recovery of its meaning from the consideration of certain tracks on the same text. Said recovery takes place, undoubtedly, by performing mental operations that runs the reader in order to make sense of the clues found. We refer to a dynamic process that develops reading consistent sets as connections between the information held in their cognitive structures and new information that provides the text.

Based on these theoretical postulates develop our proposal through the following topics: the reading process; reading phases; major conflicts and possible resolution strategies.

\section{Keywords}

Reading - Strategies - Process - Understanding Conflict

\section{Resumo}

No artigo a seguir, vamos desenvolver algumas reflexões e estratégias de resolução, ligadas a um problema que afeta a comunidade universitária será proposto: o processo de leitura e compreensão de textos acadêmicos.

Acreditamos na compreensão da leitura de um texto acadêmico como a recuperação de seu significado a partir da consideração de certas faixas do mesmo texto. Disse recuperação ocorre, sem dúvida, através da realização de operações mentais que executa o leitor, a fim de fazer sentido das pistas encontradas. Referimo-nos a um processo dinâmico que se desenvolve a leitura sets consistentes como conexões entre as informações contidas em suas estruturas cognitivas e novas informações que fornece o texto.

Com base nesses postulados teóricos desenvolver nossa proposta através dos seguintes temas: o processo de leitura; ler as fases; grandes conflitos e estratégias de resolução possíveis.
Palavras Chave

Leitura - Estratégias - Processo - Compreensão Conflito

\section{Introducción}

Cualquier lector universitario es capaz, técnicamente, de leer letra impresa; pero ¿es capaz de comprender perfectamente el texto, de comentarlo competentemente y de responder preguntas de los profesores acerca del mismo?

Estos interrogantes se plantean cuando advertimos que nuestros alumnos poseen amplias dificultades para la comprensión y comunicación del contenido de sus lecturas. Se limitan a la mera reproducción casi memorística y literal del texto, sin evidenciar un proceso de internalización y aprehensión del mismo. Esta situación, particularmente en el ámbito universitario, se transforma en un conflicto.

Algunos autores sostienen que deberíamos referirnos a lectores analfabetos en lo concerniente al sentido de la experiencia de la lectura $\circ$ a la lectura como experiencia. Asimismo, plantean una cuestión interesante cuando aluden a que tal vez los aparatos educativos -con el transcurrir de los años- han privilegiado la comprensión, puesto que tanto los discursos psicotécnicos como pedagotécnicos sobre la lectura se mueven exclusivamente en el interior del marco de la comprensión.

Ana María Finocchio desarrolla dos hipótesis al respecto:

"La primera es que en la escuela que conocemos es esencial la evaluación. Por lo tanto, es esencial hacer visibles de una forma máximamente estandarizada cuáles son los resultados de las prácticas de enseñanza, si sus objetivos han sido o no alcanzados, y de qué modo. $Y$ para eso, el modelo de la comprensión es perfecto. De lo que se trata es de saber si el alumno ha comprendido lo que tiene que comprender, y a partir de ahí enseguida podemos establecer problemas de comprensión, niveles de comprensión, y todas esas cosas que les gustan tanto a los psicopedagogos en ejercicio.

La segunda hipótesis tiene que ver con el predominio de la concepción técnica del lenguaje, esa que considera la lengua como instrumento de comunicación. La lengua no es otra cosa que un soporte de ideas, 
REVISTA FACULTAD DE ODONTOLOGÍA

ISSN No 1668-7280 - Vol. VII No 1 - 2014

47
EDUCACIÓN

La lectura en el ámbito universitario. Estrategias para intentar resolver un conflicto. sentimientos y, en general, expresiones, y leer no es otra cosa que apropiarse de eso que la lengua comunica. La lengua no es otra cosa, en definitiva, que soporte y transporte de información. No es otra cosa que telecomunicación. No creo necesario insistir en la cantidad de discursos que se asientan sobre ese supuesto. Desde la concepción cognitivista de la lectura, según la cual leer no es otra cosa que procesar información, hasta toda esa retórica de la sociedad de la información que se está imponiendo sin crítica y con el apoyo de estados y oligopolios de todo el mundo. No creo necesario insistir tampoco en la cantidad de programas de investigación educativa y de formación del profesorado que incluyen una $u$ otra de esas retóricas. El sistema educativo trabaja el lenguaje desde el punto de vista de la tecnología de la información. Por eso trabaja la lengua desde el punto de vista de su máxima transparencia y de su máxima eficacia."'

En el siguiente trabajo se expondrá una problemática imperante en el ámbito universitario y se propondrán algunas estrategias para intentar responder parcialmente a un conflicto que atraviesa a todo el ámbito académico.

\section{Desarrollo}

\section{El proceso de la lectura}

Pensamos en la comprensión de la lectura de un texto académico como la recuperación de su significado a partir de la consideración de ciertas pistas contenidas en el mismo texto. La mencionada recuperación se lleva a cabo -indudablemente- mediante la ejecución de operaciones mentales que pone en funcionamiento el lector con la finalidad de darle sentido a las pistas halladas. Referimos a un proceso dinámico que desarrolla el que lee a medida que establece conexiones coherentes entre la información que posee en sus estructuras cognitivas y la nueva información que le provee el texto.

De acuerdo con esta línea de pensamiento, hablamos de una interacción analítica y dinámica entre la información de la que dispone el lector acerca del lenguaje y del contenido del texto, junto con la información suministrada por el propio texto.

I Material de clases Especialización en Lectura, Escritura y Educación. FLACSO, 2010.
Enmarcándonos en los enfoques interactivos hablamos de que la concepción a la que adherimos respecto de la lectura es de tipo procesual; es decir, que acordamos con un procesamiento que deriva de la interacción lector-texto-contexto de forma dinámica y en la que el lector asume un rol activo, crítico y creativo -rol que lo lleva a desarrollar una serie de estrategias que colaboran con el procesamiento de la información.

De esta manera, se concibe que el lector -para ser un buen entendedor- cuenta con un amplio bagaje de estrategias tanto generales como específicas, a los fines de poder seleccionar aquellas que resulten adecuadas y eficaces para cada situación, para cada modalidad de lectura y para cada texto.

Peronard (1993) ha observado que en estas últimas décadas hubo un cambio en relación con el rol activo del lector enfrentado a un texto, el que se debe -en parte- al auge de las ciencias cognoscitivas las cuales, usando la computadora como modelo del cerebro humano, conciben a éste como un versátil procesador de información.

De acuerdo con este marco teórico, el lector aborda el texto a partir de una cultura general, de una cultura sobre su lengua y de una cultura de la comunicación escrita. Consecuentemente, la experiencia previa desempeña un lugar fundamental en la construcción del significado. Durante ese proceso de construcción, el lector va anticipando y formulando predicciones acerca de diversos aspectos del texto, los que luego va a ir confirmando a través de la lectura (Goodman, 1985). Así, produce diversas reelaboraciones desde el inicio hasta el final del proceso de lectura.

En síntesis, la lectura es un proceso global en el cual el lector está preocupado por lograr el sentido del texto.

\section{Las fases de la lectura}

Para Flood y Lapp (199I), los lectores no sólo tienen un plan para comprender sino que usan sus conocimientos metacognitivos de forma ordenada para implementar su plan. Estos autores sostienen que, a pesar de los ajustes requeridos por la variación textual, los lectores estratégicos 
presentan los siguientes pasos en su plan general:

- Antes de leer, anticipan el texto a través de los paratextos que acompañan al mismo, construyen un trasfondo esquemático activando los conocimientos previos apropiados y determinan sus propósitos para leer.

- Durante la lectura, comprueban el entendimiento del texto o elaboran hipótesis de lectura (Goodman, 1995; Iser, 1975), regulan su comprensión empleando claves contextuales e integran los conceptos extraídos del texto con los ya existentes en sus esquemas mentales.

- Después de leer, los lectores estratégicos resumen, evalúan y aplican las ideas presentes en el texto leído.

Estas fases se corresponderían con las siguientes:

1. PRELECTURA: En esta fase la intención radica en orientar al lector para abordar el texto. Se pueden emplear diversas estrategias para acompañar esta fase, como ser:

- Ubicar al lector en el contexto del texto: considerar las construcciones del enunciador $y$ el enunciatario (quién "habla" y cómo y a quién se dirige) (Arnoux, E, 20I3).

- Proponer al estudiante algunas preguntas que tendrá que tener en mente mientras lee.

- Acompañar al texto con un glosario.

2. LECTURA: Esta fase se corresponde con el proceso propiamente dicho de la lectura del texto. Es factible acompañar al texto con un cuestionario de comprensión lectora.

3. POSLECTURA: En esta fase la intención se fundamenta en constatar cuál fue el proceso de lectura que ha efectuado el lector. Algunas estrategias pueden vincularse con:

- Repasar y reforzar el vocabulario del texto.

- Presentar ejercicios en los que se practiquen estrategias de generación de ideas, llevando al estudiante a establecer una generalización a partir de datos concretos que proporciona el texto.

\section{Principales conflictos}

Nuestra experiencia en el ámbito universitario nos ha permitido identificar los siguientes conflictos en la comprensión lectora de textos académicos:

- Conflictos para ingresar en el texto en tanto que unidad de significados relacionales. Esta cuestión se evidencia por una ausencia de referentes, lo que indica una lectura localizada en las formas de lenguaje más no en las relaciones de significado que se establecen en la continuidad semántica.

- Conflictos para interactuar con la propuesta de organización textual realizada por el autor del texto. Esto se reconoce porque el lector efectúa una lectura basada exclusivamente en sus esquemas personales.

- Conflictos para reconocer las ideas más importantes que engloban la información del texto y la forma en las que el escritor las ha puesto en relación a través de una estructura retórica determinada.

- Conflictos para comprender los contextos situacionales, la situación de comunicación que genera el texto y que viabiliza identificar los propósitos del autor en relación con el lector, como ser: informar, describir, convencer, persuadir, explicar.

- Conflictos para alejarse del texto y autorregular su proceso de comprensión.

De esta manera, consideramos que son pocos los estudiantes que han alcanzado -a través de diferentes niveles de instrucción- el desarrollo y manejo de estrategias discursivas que les permitan comprender los textos de divulgación y menos aún los académicos -en sus diferentes fases de complejidad léxica y disciplinar-.

\section{Posibles estrategias de resolución}

Si bien no creemos en las recetas mágicas, sí estamos seguros de que la implementación de algunas estrategias puede contribuir con la resolución de ciertos conflictos.

De alguna manera lo hemos planteado a lo largo del desarrollo del presente artículo cuan- 
do sostenemos que, para un proceso verdaderamente eficaz, reflexivo y crítico vinculado con la lectura, es recomendable que el lector asuma el control y la evaluación de su propio proceso de comprensión (estrategias de metacomprensión). Teniendo en cuenta que la lectura es una actividad compleja constituida por diferentes procesos cognitivos, es factible que al verse afectado alguno de ellos se obstaculice la comprensión.

Varias investigaciones han demostrado que existen diferencias marcadas entre lectores eficientes y lectores no eficientes. Dentro de los conflictos y deficiencias más usuales encontramos:

I) Escasa o nula conciencia de que deben hallar sentido en lo que leen, dicho en otros términos, no asumen que leen con el objetivo de construir el significado del texto. Esta cuestión se evidencia porque no pueden confrontar los contenidos del texto con sus saberes previos, ni son conscientes de lo importante y necesarias que son las inferencias durante el proceso de la lectura y se restringen a la mera decodificación del texto.

2) Escasa o nula autoevaluación de su proceso de lectura; es decir, muchas veces no advierten que no han comprendido el texto. Recordemos que leer no es comprender.

3) Escaso o nulo empleo de estrategias compensatorias para resolver dificultades en la comprensión. En general, los lectores no eficientes no participan ni activa ni estratégicamente en el proceso lector, no toman conciencia del proceso ni lo asumen como tal. Por el contrario, el lector eficiente (Garner, 1980) se relaciona e interactúa con el texto, tiene conciencia y asume el control de las tareas que ejecuta durante el proceso de lectura; asimismo, hace uso de estrategias que se ajustan a sus propósitos de lectura, a la tarea específica que el texto le demanda y al tipo de texto con el que se enfrenta.

Un proceso estratégico de comprensión de textos requiere de ciertos procedimientos específicos orientados a desarrollar las diversas operaciones responsables de la comprensión y de su control para lograr un proceso eficaz. Se trata de alcanzar metas concretas, haciéndolo de manera óptima a través de acciones específicas orientadas a esos fines.

Este proceso permite mejorar el desempeño y generar conocimiento nuevo en los lectores. La denominada autorregulación del conocimiento y la construcción de conocimientos aparecen como aspectos indisociables de la estructura de conocimiento de cualquier individuo.

De esta manera, las estrategias de lectura que se proponen desde la metacognición por varios especialistas se corresponden también con estrategias cognitivas, como ser:

- El establecimiento de metas y propósitos de lectura

- La activación de esquemas cognitivos adecuados

- La generación y comprobación de expectativas, predicciones e hipótesis de lectura

- La focalización selectiva de la atención sobre la información esencial

- La elaboración y comprobación de inferencias

- La elaboración de esquemas y resúmenes

Baker y Brown, 198I; Brown y otros, 1984, 1986; Garner, 1987; Palincsar, 1993; Paris y otros, 1983; proponen algunas estrategias de metacomprensión que nos resultan interesantes para considerar en esta instancia de buscar posibles soluciones a un conflicto instaurado respecto de la lectura en el ámbito universitario. Estas estrategias se agrupan en torno a los que se pueden considerar como los dos objetivos básicos de la metacomprensión: la toma de conciencia de la lectura como proceso estratégico y el autocontrol y la autorregulación de todo el proceso.

La toma de conciencia del proceso lector:

- Tener en claro el propósito de la lectura, a los fines de poder anticipar las demandas que requiere la tarea

- Planificar el proceso de lectura

- Focalizar selectivamente la atención en lo esencial del texto 
- Reconocer la estructura del texto para verificar las interrelaciones que se producen entre sus componentes.

El control y la regulación del proceso:

- Tomar notas en relación con la información relevante del texto, por ejemplo a través de la confección de esquemas de contenido.

- Construir mapas conceptuales y cuadros sinópticos.

- Elaborar una síntesis de los contenidos con un objetivo de escritura preciso. A diferencia del resumen, que mantiene una fidelidad casi literal respecto del texto base, la síntesis permite una reformulación personal de los conceptos esenciales del texto, con un vocabulario y estilo propios.

- Explorar los significados de los elementos léxicos del texto.

- Analizar el significado de los conectores presentes en el desarrollo de las explicaciones más relevantes del texto para entender la lógica que se establece entre las ideas.

- Considerar el contexto como un aspecto importante en la construcción del sentido: ¿quién es el autor? ¿qué se propone con el texto? ¿qué nos quiere decir sobre el tema?

- Decidir acerca de la necesidad o conveniencia de tomar acciones correctivas o compensatorias en caso de que fuera necesario. Esto es importante ya que supone la posibilidad de tener problemas con la comprensión e implica poder identificarlos y decidir qué hacer al respecto.

\section{Conclusión}

Consideramos que las estrategias expuestas, abordan, desde una perspectiva diferente, los conflictos lectoescriturarios predominantes en los alumnos universitarios con los que usualmente trabajamos. Asimismo, que estas estrategias implican pensar en las prácticas más allá de la mera comprensión -sea esta parcial o total-, y contemplarlas como posibles resultantes del encuentro del lector con el texto, entendiendo este acto en su cabal complejidad.
En síntesis, significaría pensar estas cuestiones desde un espacio en el que tanto lector como texto interactúan, en las que conocemos cuáles son los fines de la lectura (para qué se lee y en qué circunstancias se lo hace) más allá de concebir el texto como mero "llenado" de información. Esto último es de fundamental importancia para situar las prácticas de lectura $-y$ de escritura- en el ambiente universitario. Paula Carlino ha entendido que las unidades académicas constituyen un universo donde éstas prácticas cambian y constituyen otro discurso, una nueva cultura. Desde este punto de vista, es importante entender que los alumnos no "comprenden" sólo trasladando sus hábitos lectores a la universidad. 
REVISTA FACULTAD DE ODONTOLOGÍA

ISSN No 1668-7280 - Vol. VII № 1 - 2014

51
EDUCACION

La lectura en el ámbito universitario. Estrategias para intentar resolver un conflicto.

\section{Bibliografía}

I. Arnoux, E. et al. (2013) La lectura y la escritura en la Universidad. Eudeba, Bs. As.

2. Baker, L. Y Anderson, R. C. (1984) Cognitive monitoring in reading. J. Flood (ed.), Understanding Reading Comprenhension, I. R. A., Newark, Delaware.

3. Baker, L. Y Brown, A. L. (198I) Metacognition and the reading process. P. D. Pearson y otros (Ed.), Handbook of Reading Research, Longman, London.

4. Brown, A. L. y otros (1984) Instructing comprenhension fostering activities in interactive learning situations. H. Mandl y otros (ed.) Learning and Comprenhension of Text, Lawrence Erlbaum Assoc., N. J.

5. (1986) The role of metacognition in reading and studying. J. Orasanu (ed.) Reading Comprenhension from Research to Practice, Erlbaum, N. J.

6. Bernárdez, E. (1982) Introducción a la lingüística del texto. Madrid, Espasa - Calpe.

7. Conti De Londero, M. Y Sosa De Montyn, S. (2010) Hacia una gramática del texto. Córdoba, Comunicarte.

8. Dubois, M. E. (1987) El proceso de lectura: de la teoría a la práctica. Buenos Aires, Grupo Aique.

9. Flood, J. Y Lapp, D. (1991) Reading comprenhension instruction. En: Flood, J.; Jensen, J.; Lapp, D. y Squire, James (Eds.), Handbook of research on teaching the English language arts, New York, Macmillan Publishing Company.

I0. Garner, R. (1980) Monitoring of understanding. J. of Reading Behavoir, 12.

II. ------------ (198I) Monitoring of passage inconsistencies among poor comprehenders. J. of Educational Research 74.
12. Goodman, K. (1985) On being literate in an age of information. En Journal of Reading, Vol. 28, $\mathrm{N}^{\circ}$ 5.

13. Iser, W. (972) "El proceso de lectura: una aproximación fenomenológica" en Mayoral, J. A. (Comp.) Estética de la recepción. Arco, Madrid, Pp. 2II5-243

14. Martínez, M. C. (Comp.) (1997) Los procesos de la lectura y la escritura. Santiago de Cali, Editorial Universidad del Valle.

15. Morles, A. (1986) Entrenamiento en el uso de estrategias para comprender la lectura. En: Revista Latinoamericana de Lectura y Vida. Año $7, \mathrm{~N}^{\circ}$ 2; pp. 15-20.

16. Palincsar, A. S. (1983) Becoming a strategic reader. Contemporary Educational Research, 8.

17. PARIS, S. G. y otros (1988) Models and metaphores of learning strategies. C. E. Weinstein y otros (ed.) Learning and Studying Strategies. Academic Press, N. Y.

18. Peronard, M. Y Uriz, N. (1990) La enseñanza de estrategias para la comprensión lectora. Signos; Vol. XXIII, N²8, pp. I09-II8.

19. Pipkin Embón, M. Y Reynoso, M. (2010) Prácticas de lectura y escritura académicas. Córdoba, Comunicarte.

20. Puente, A. (199I) Comprensión de la lectura y acción docente. Madrid, Ediciones Pirámide S. A.

2I. Ruddell, A. (|99|) Comprensión de la lectura y acción docente. Madrid, Ediciones Pirámide S. A.

22. Smith, F. (1983) Comprensión de la lectura. Análisis psicolingüitsico de la lectura y su aprendizaje. México, Trillas. 